\title{
A Biologically Inspired Model for the Detection of External and Internal Head Motions
}

\author{
Stephan Tschechne, Georg Layher, and Heiko Neumann \\ Institute of Neural Information Processing, University of Ulm, \\ 89069 Ulm, Germany \\ http://www.uni-ulm.de/in/neuroinformatik.html
}

\begin{abstract}
Non-verbal communication signals are to a large part conveyed by visual motion information of the user's facial components (intrinsic motion) and head (extrinsic motion). An observer perceives the visual flow as a superposition of both types of motions. However, when visual signals are used for training of classifiers for non-articulated communication signals, a decomposition is advantageous. We propose a biologically inspired model that builds upon the known functional organization of cortical motion processing at low and intermediate stages to decompose the composite motion signal. The approach extends previous models to incorporate mechanisms that represent motion gradients and direction sensitivity. The neural models operate on larger spatial scales to capture properties in flow patterns elicited by turning head movements. Center-surround mechanisms build contrast-sensitive cells and detect local facial motion. The model is probed with video samples and outputs occurrences and magnitudes of extrinsic and intrinsic motion patterns.
\end{abstract}

Keywords: Social Interaction, HCI, Motion Patterns, Cortical Processing

\section{Introduction}

The recent development of computers show a clear trend towards companion properties $[4,14]$. Systems are demanded to improve the efficiency of human computer interaction (HCI) by adapting to the user's need, experience and moods. To achieve this, those systems must be able to interpret non-verbal communication patterns that are signalled by the user $[2,5]$. These patterns are to a large part contained in visual signals that can be captured by an interaction system, from which they can automatically be derived [9]. Among these visual patterns bodily and facial expressions contain important cues to emotionally relevant information, whereas both types can be either of static or dynamic nature. Optic flow has been investigated for emotion and facial analysis earlier $[13,6,11,8]$. However, when it comes to analysis of facial motion, extrinsic (head) and intrinsic (facial) movements are superpositioned and elicit a composite flow field of respective patterns from the observer's perspective (see Fig. 1). Subsequent classification stages profit from a segregation of these patterns. In [13], an attempt to decompose the facial optical flow into affine as well as higher-order 
flow patterns in order to segregate the facial motion has been proposed. In [3] head-pose and facial expressions are estimated graphics and animation. Here, an affine deformation with parallax is modelled to fit active contours using singular value decomposition. In [1] the authors propose a multi-stage landmark fitting and tracking method to derive face and head gestures.

In this paper we propose a novel mechanism to detect occurrences of basic components of motion from optic flow. The method is studied on the example of head movements and dynamic facial expressions which both cause optic flow at the observer position. We model mechanisms of signal processing in early and intermediate stages of visual cortex to provide robust automatic decomposition of extrinsic and intrinsic facial motions. We demonstrate how occurrences of extrinsic as well as intrinsic components are robustly derived from an optic flow field. Our approach contrasts others by neither requiring face detection or a previous learning phase. Additionally, processing of multiple persons comes at no extra cost.

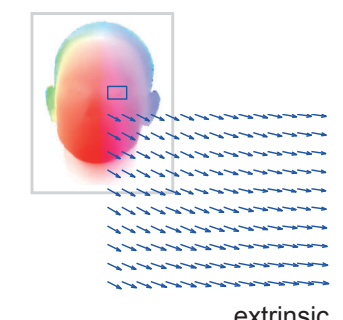

extrinsic
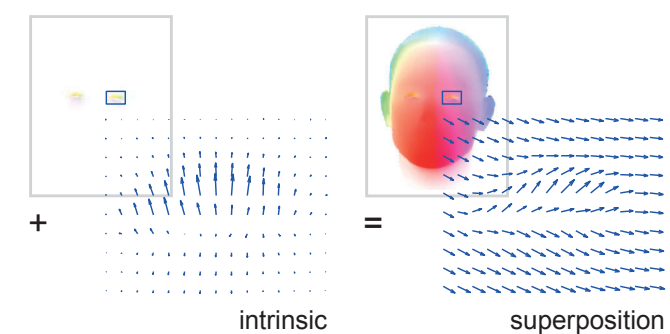

Fig. 1. Visual flow at the observer position is a superposition (right) of extrinsic (head, left) and intrinsic (facial, middle) motion. Subsequent processing benefits from separated processing of both sources.

\section{Visual Representations of Head Movements}

The instantaneous motion of a three-dimensional surface that is sensed by a stationary observer can be represented by the linear combination of its translational and rotational motion components [10], as well as non-rigid motion caused by object deformations. Any of these cause visual motion in the image plane. In this paper we focus on the analysis of facial and head motion of an agent in a communicative act by means of optic flow. This motion is composed of the extrinsic (head) motion caused by translations and rotations and the superimposed internal motion of facial components (intrinsic motion). We assume that any translational extrinsic motion of the head has been compensated to fixate the head in the middle of the image so that the world coordinate system is centered in the moving head. We aim at spatial processing of the resulting patterns 
to individually detect the extrinsic and intrinsic components. For a rotation of a simple head model around the $Y$-axis, an arbitrary surface point $P=(x, y, z)^{T}$ appears on a rotational path in space (see Fig.2) with frequency $\omega$ and periodic length T. $P$ is uniquely defined by its radius $r$ and vertical component $y$ at time $t$, when a distance $d$ to the observer is assumed:

$$
P(t, r, y)=r \cdot\left(\begin{array}{c}
\cos \omega t \\
y \\
\sin \omega t
\end{array}\right)-\left(\begin{array}{l}
0 \\
0 \\
d
\end{array}\right) \quad \omega=\frac{2 \pi}{T}
$$

In the following we assume that $y=0$ and $r=1$. Application of the projection model with $x=f \cdot X / Z$ and $y=f \cdot Y / Z$, given the focal length $f$ of the camera yields the projected coordinates $P_{\text {proj }}$ in image plane for the observer:

$$
P_{\text {proj }}(t, r)=f \cdot\left(\begin{array}{c}
\frac{r \cos \omega t}{r \sin \omega t-d} \\
0
\end{array}\right)
$$

If we assume constant angular speed, the apparent image speed of the projection of $P$ is

$$
\frac{\partial P_{p r o j}(t, r)}{\partial t}=\left(\begin{array}{c}
\frac{r \sin \omega t+d-1}{r^{2} \sin ^{2} \omega t-2 d r \sin \omega t+d^{2}} \\
0
\end{array}\right)
$$

which yields a characteristic motion pattern, see Fig. 2. The apparent motion on the positive half circle, where the facial surface is oriented towards the observer, leads to a generic speed pattern. For a frontal motion from right to left the pattern is composed by an acceleration, followed by maximum frontal motion, and a symmetric deceleration patch. This pattern corresponds to the speed gradients as investigated by [12] and is also depicted in Fig. 2. For symmetric and bounded objects image patches of increasing and decreasing speeds are juxtaposed reflecting appearance and disappearance of the surface markings on a convex rotating body surface. In Sec. 2.1 we suggest a filtering mechanism for these arrangements of speed gradients which is tuned to such symmetric arrangements of image motions with symmetric speed changes.

Facial motion, on the other hand, is caused by actions of facial muscles, e.g. during verbal communication, eye blinks, or while forming facial expressions. These spatio-temporal changes occur as deformations on a smaller temporal and spatial scale compared to the size of the face and are characterized by changes in motion direction and/or speed relative to its coherent surrounding motion. In order to analyze such intrinsic facial motions, we reasoned that a centersurround mechanism for the filtering of motion patterns within the facial region will indicate occurrences of intrinsic motions, see Sec. 2.2.

\subsection{A Model of Cortical Motion Gradient Detection}

In the following we describe the implementation details of our model cells for detecting motion patterns that are characteristic for extrinsic motions corresponding to rotations around the $X$ - or $Y$-axis, respectively, namely patterns 

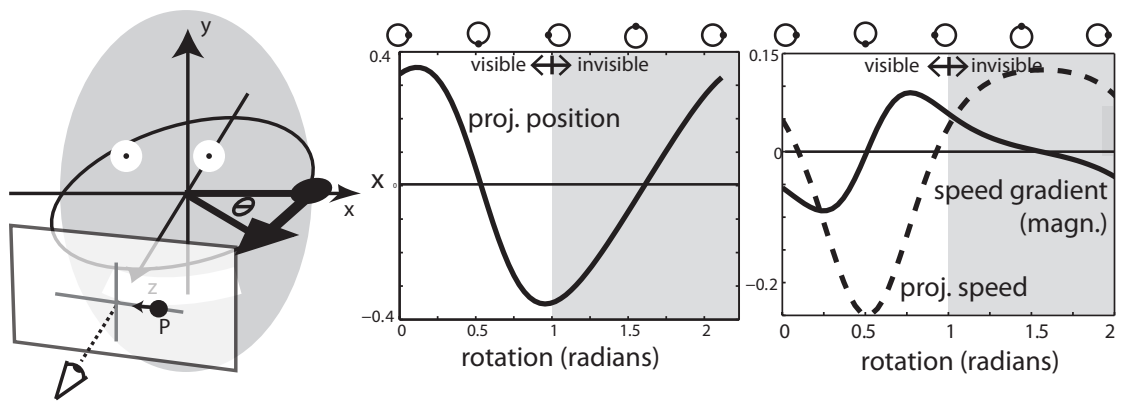

Fig. 2. Left: Projection model of one point on a head's surface and its trajectory in the projection when the head is rotating. Middle: X-position over rotation angle of point P. Right: One-periodical plot of speed over rotation angle (dashed) and speed gradient (solid) of a point when rotating on a circular path around $Y$ axis. Point $P$ is closest at position 1.0. Due to foreshortening effects, characteristic speed gradients occur where the point approaches or retreats.

containing speed gradients. All presented detectors need a visual motion field $\mathbf{u}$ which is transformed into a log-polar representation, the velocity space $\mathbb{V}$. In velocity space, motion is separably represented by speed $s_{p}=\log \|\mathbf{u}\|$ and direction $\tau_{p}=\tan ^{-1}\left(\frac{u_{y}}{u_{x}}\right)$. This representation allows selecting image locations containing certain motion directions, speeds, or both, which will be fundamental features for the upcoming design of gradient cells. Filters for speed $\psi$ and direction $\theta$ with tuning widths $\sigma$ are defined by

$$
\begin{aligned}
& F_{S}(\mu, \nu)=\exp \left(-\frac{(\mu-\psi)^{2}}{2 \sigma_{\psi}^{2}}\right) \\
& F_{\theta}(\mu, \nu)=\exp \left(-\frac{(\nu-\log (\theta))^{2}}{2 \sigma_{\theta}^{2}}\right) \forall(\mu, \nu) \in \mathbb{V} .
\end{aligned}
$$

Gradient cells $M_{p}^{+/-}$at image position $p$ respond when two conditions are served, see Fig. 3: First, conjunctive input configurations need to match their tunings for speed and directions, and second, an increase or decrease in speed along an axes corresponding to their directional exists. This increase or decrease is reflected in a simultaneous stimulation of two speed- and direction-tuned cells that are spatially arranged to build the desired speed gradient. The speed- and direction-tuned subcells $M_{p}$ are derived from the given motion field by applying a filter $F$ in velocity space representation. Each cell response incorporates a divise normalisation component in order to keep responses within bounds.

$$
\begin{aligned}
M_{p} & =\mathbb{V}_{p} \cdot F \\
M_{p}^{+} & =M_{p-\Delta p} \cdot M_{p+\Delta p} \cdot\left(\epsilon+M_{p-\Delta p}+M_{p+\Delta p}\right)^{-1} \quad(0<\epsilon \ll 1)
\end{aligned}
$$


Responses to flow gradients of opposite polarity are subsequently combined by $A N D$-gating to build a curvature detector. These cells operate at spatially juxtaposed locations with component offsets along their directional preference depending on the spatial scale of the filter kernels.

$$
C_{p}=M_{p+\Delta p}^{+} \cdot M_{p-\Delta p}^{-} \cdot\left(\epsilon+M_{p+\Delta p}^{+}+M_{p-\Delta p}^{-}\right)^{-1}
$$

In order to make the response more robust and selective to motion direction this curvature response is combined with the output of a motion integration cell $M_{p}$. The final response is thus defined by

$$
R_{p}=M_{p+\Delta p}^{+} \cdot M_{p} \cdot M_{p-\Delta p}^{-} \cdot\left(\epsilon+M_{p+\Delta p}^{+}+M_{p}+M_{p-\Delta p}^{-}\right)^{-1}
$$

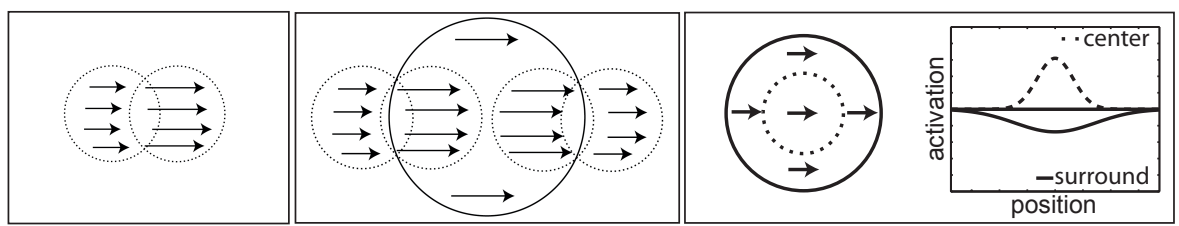

Fig. 3. Left: Gradient Cell $M p^{+}$. Middle: The full model cell circuit for detecting rotational motion patterns. Oppositely tuned gradient cells are combined with cells sensitive to uniform motion patterns. Right: Center-surround cell for motion discontinuity detection with two centered subcells with different spatial tunings and integration weights for center and surround cell.

\subsection{Model Mechanisms for Motion Contrast Detection}

Local facial motions can be accounted by mechanisms that operate on a smaller scale within the facial projection into the image plane. To detect intrinsic motion, we propose cells that are sensitive to local changes in speed and direction. These motion patterns are produced in the facial plane while the person is talking or during other facial actions. Here, we employ a center-surround interaction mechanism of motion sensitive cells that are able to detect local variations in visual flow, but won't be sensitive to large uniform flow fields. Such a sensitivity can be generated by cells with antagonistic center-surround motion sensitivity. The input integration of velocity responses is defined by weighted kernels $\Omega$ with different spatial scale dimensions operating on responses of motion and speed selective filters. Integration over $N$ directions yields the activation for a direction-insensitive motion contrast cell.

$$
D_{p, \theta}^{s u b}=\mathbb{V}_{p} * F_{\bar{\mu}}
$$




$$
\begin{gathered}
D_{p, \theta}=\frac{\max \left(0, D_{p, \theta}^{\text {sub }} * \Omega_{\text {inner }}-D_{p, \theta}^{\text {sub }} * \Omega_{\text {outer }}\right)}{\epsilon+D_{p, \theta}^{\text {sub }} * \Omega_{\text {inner }}+D_{p, \theta}^{\text {sub }} * \Omega_{\text {outer }}} \\
D_{p}=\sum_{\theta \in n}\left\|D_{p, \theta}\right\| \quad n=\{1, \ldots, N\}
\end{gathered}
$$

\section{Results}

Our model was probed with short video sequences containing extrinsic or intrinsic motion. Optic flow was generated from these scenes by using a high quality estimation method [7], see top four images of Fig. 4. The optic flow was then transformed into velocity space representation and presented to proposed model cells. The middle section of Fig. 4 shows results for sequences of unconstrained motion, where persons could move the head ad libitum. The persons were told to blink with their eyes randomly to simulate small intrinsic motions. Head rotation and eye blinks were manually annotated. Extrinsic motion was then detected by the proposed model cells. Plots of the activations are also shown in the figure. The rotation of the head is recovered and a differentiation is made between leftward and rightward motion. Also, detectors for intrinsic motion show activations that correlate well with eye blink labels. Responses of model cells were temporally filtered with a moving average operation to eliminate spurious temporal artefacts due to errors in the flow estimation process. Note that the detectors not only indicate the presence of an occurrence but also the position. This also holds when a sequence with multiple persons is processed, see bottom four images of Fig. 4.

\section{Conclusion and Discussion}

We propose a biologically inspired model of motion processing that is sensitive to occurrences of motion patterns specifically produced by extrinsic or intrinsic head motions in user interaction scenarios. These movements are perceived as composite motion signals by the observer. Correct interpretation regarding user dispositions and non-verbal communication patterns from visual signals requires segregated processing of both sources. We propose networks of cortical motion processing to detect the individual occurrences of intrinsic and extrinsic motion. The model incorporates a stage of detectors for rotations around $X-$ and $Y$ - axes that include sensitivity to motion gradients and motion direction. Center-surround mechanisms indicate intrinsic motions by detecting local contrasts that are produced by local motions of facial components. We probed the model with realistic input sequences containing head rotations and eye blinks to represent both motion components. Both proposed detectors work well on facial images and segregate composite facial motion into their extrisinic and intrinsic components. Our approach does not need training or face detection and can 


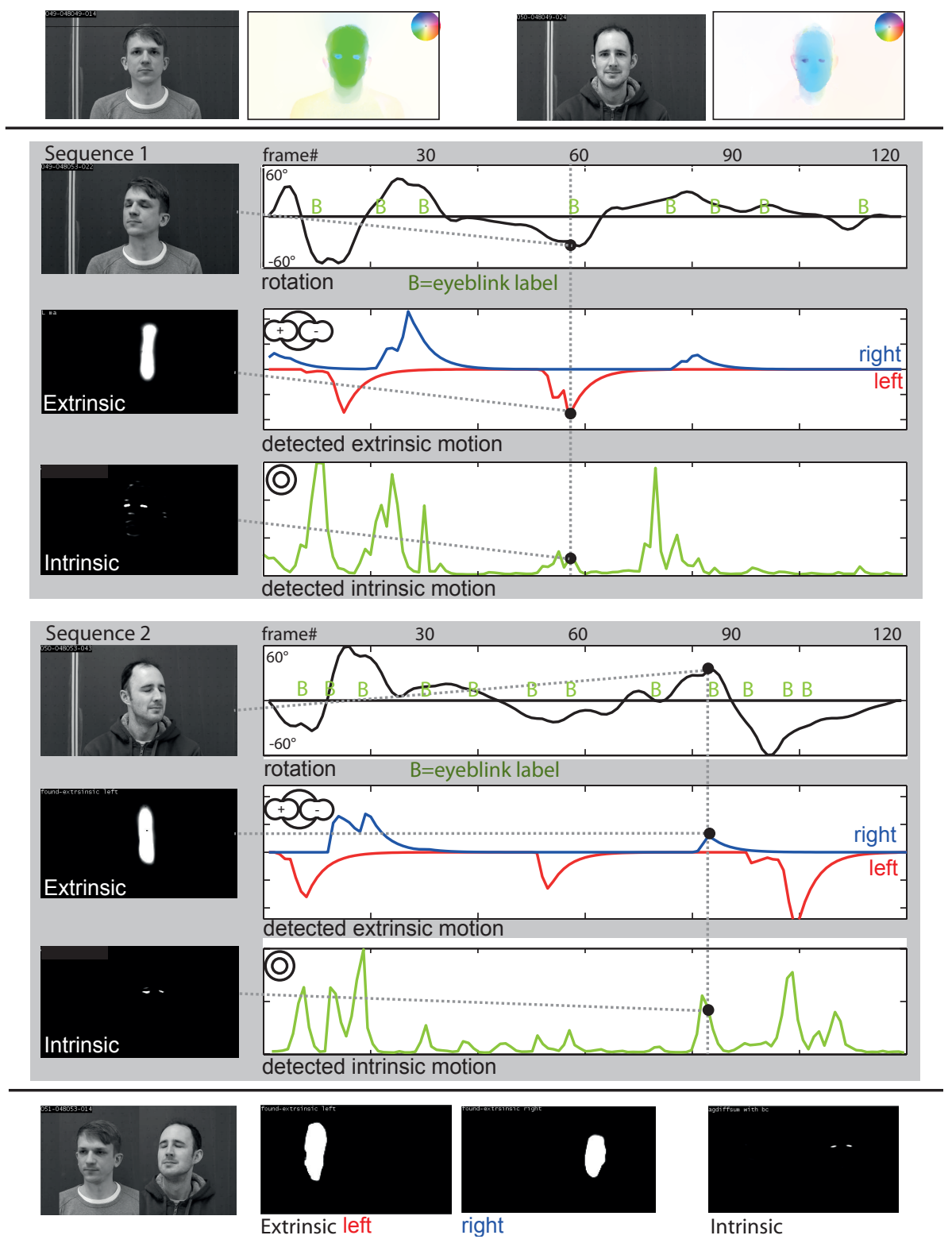

Fig. 4. Results for the processing of two interaction sequences with unconstrained head motion. Top four images: Sequence snapshots and color-coded optic flow fields. Large central part: Manually labeled head rotation and eye blinks (1st\&4th plot), activation of rotation-selective cells (2ndE5th plot) and activation of cells for intrisic motion (3rdE6th plot). Single processing snapshots are arranged on the left, their points of reference are indicated by dotted lines. Bottom four images: Snapshot of synthesized video with a test sequence containing two persons to demonstrate simultaneous processing. In the selected snapshot the two persons rotate their head differently, which is reflected in the activations of respective cells. The right person additionally blinks with his eyes. 
process multiple persons in a sequence simultaneously. Subsequent processing steps profit from the detection of occurrence and position of movement components. In contrast to other approaches our proposed model is independent from highly specialised models, tracking, learning from examples or large optimisation stages to derive the presented results. By this we demonstrate how human-computer-interfaces profit from biologically inspired mechanisms of motion processing by detecting occurrences of specific motion patterns from an optic flow field. Future work will include a validation of the approach for more generic shape-from-motion tasks.

This work has been supported by grants from the Transregional Collaborative Research Center SFB/TRR62'A Companion Technology for Cognitive Technical Systems' funded by the German Research Foundation (DFG).

\section{References}

1. H C Akakin and B Sankur. Robust Classification of Face and Head Gestures in Video. Image and Vision Computing, 29:470-483, 2011.

2. M Argyle. Bodily Communication, volume 2. Routledge, London, 1988.

3. B Bascle and A Blake. Separability of Pose and Expression in Facial Tracking and Animation. In Proc. Int. Conf. on Computer Vision, pages 323-328, 1998.

4. D Benyon. Designin Interactive Systems, 2nd Ed. Pearson, Addison-Wesley, London, 2010.

5. D Benyon and O Mival. Landscaping Personification Technologies: From Interactions to Relationships. In CHI Ext. Abs. on Human Factors in Computing Systems, pages 3657-3662, 2008.

6. M Black and Y Yacoob. Recognizing Facial Expressions in Image Sequences Using Local Parameterized Models of Image Motion. International Journal of Computer Vision, 25(1):23-48, 1997.

7. T Brox, N Papenberg, and J Weickert. High Accuracy Optical Flow Estimation Based on a Theory for Warping. In European Conference on Computer Vision (ECCV), volume 3024, pages 25-36. Springer, 2004.

8. G Donato, M S Bartlett, J C Hager, P Ekman, and T J Sejnowski. Classifying Facial Actions. IEEE Transactions on Pattern Analysis and Machine Intelligence, 21(10):974-989, 1999.

9. C D Frith and D M Wolpert. The Neuroscience of Social Interaction: Decoding, imitating, and influencing the actions of others. Oxford University Press, 2004.

10. H C Longuet-Higgins. A Computer Algorithm for Reconstructing a Scene from Two Projections. Nature, 293:133-135, 1981.

11. R Niese, A Al-Hamadi, A Farag, H Neumann, and B Michaelis. Facial Expression Recognition Based on Geometric and Optical Flow Features in Colour Image Sequences. IET Computer Vision, pages 1-11, 2011.

12. G Orban. Higher Order Visual Processing in Macaque Extrastriate Cortex. Physiology Review, 88(88):59-89, 2008.

13. M Rosenblum, Y Yacoob, and L S Davis. Human Expression Recognition from Motion Using a Radial Basis Function Network Architecture. IEEE Transactions on Neural Networks, 7(5):1121-38, 1996.

14. A Wendemuth and S Biundo. A Companion Technology for Cognitive Technical Systems. Cognitive Behavioural Systems, 7403:89-103, 2012. 